\title{
Classifying Trajectories on Road Network using Neural Network
}

\author{
Deepak S. Gaikwad \\ PG student, Computer Department \\ Smt. Kashibai Navale College of Engineering, \\ Pune
}

\author{
Usha A. Jogalekar \\ Assistant Professor, Computer Department \\ Smt. Kashibai Navale College of Engineering, Pune
}

\begin{abstract}
Classification is very important in the process of Machine Learning and Data Mining. Traditional Neural Network classifier work with many kinds of data such as items, text documents, signals, networks, but there is lack of study on Trajectory Classification based on Neural Network. In this paper, proposing a system for classification of Trajectories on Road Network using classifier Neural Network. In this paper explored classification technique used for Trajectory Classification. The best feature candidate for classifying trajectory on road network is Sequential Pattern, as it preserves order of visiting sequence of Trajectories on road network. In this paper, here proposing a model using sequential pattern and neural network for acquiring high accuracy and efficiency for Trajectory Classification.
\end{abstract}

\section{General Terms}

Pattern Mining, Sequential Pattern, Neural Network.

\section{Keywords}

Classifier, Trajectory Classification.

\section{INTRODUCTION}

In recent years, many advanced technologies such as Sensors, Camera's, RFID, and GPS etc. have been developed for storing and recording information about moving objects. Analysis of data is being widely recognized [1] [2] [3] [4] [5] [6]. Trajectory classification is having various applications such as traffic prediction, city traffic planning, activity monitoring of suspicious vehicle.

This paper deal with the trajectory classification using neural network. Classifying Trajectory means that the classifying the moving object on their class label. In Trajectory Classification, (Frequent) Sequential pattern [7] [8] are good feature candidate since they preserve the order of information of trajectories [1]. While building the classification model here use the sequential pattern as a feature candidate which is extracted from data set. This feature Vector is fed to the classifier (i.e. neural network) with weight vector during Training as well as classification. The data sets used is Synthetic data, generated by the network based data generator by Brinchoff [6] as it generates the synthetic trajectories on real world road network [1].

The goal of this paper includes:

- Focusing on Trajectory Classification.

- Use of partial sequential pattern [1] for building classification model.

- Propose that the classifier plays important role while achieving high efficiency as well as accuracy.
- Intend to focus on the neural network as a best classifier for trajectory classification.

The rest the of paper is organized as follows: Section 2 Discusses the Literature Survey. Section 3 presents our proposed system. Finally Section 4 gives Conclusion and Future Work.

\section{LITERATURE SURVEY}

There are different frequent patterns based classification methods developed for different domains. Lodhi et al. [9], Lesli et. al [10], Deshpande et. al[11] have proposed classification models for text document, protein sequence, and graph respectively, they uses phrases, substring and subgraphs as a feature candidate.

Hidden Markov Model (HMM) [12] is used by Fraile and Maybank [13] for classifying vehicles trajectory. This method depends upon vehicle's motion and the sequence of internal states and it is observed that the behavior depend upon highest probability. Zheng et al. [5] have proposed the method intersecting locations and travel sequence from trajectories which enables GPS device. This model provides the users travel experience. This work is also important in trajectory pattern mining.

Mining Long, Sharable Pattern (LSP) are the methods proposed by Gidofalvi and Pedersen [2]. These LSP are not better for traffic classification as they are extremely long and having performance issue.

Jae-Gil Lee [1] proposed the framework for trajectory classification based on frequent pattern based classification. By analyzing the behavior of the trajectories on road network, it is important that the order should be preserved with the visited location. This method pretends that partial sequential pattern allow significantly improve high efficiency without losing accuracy as they preserve order as well as location of trajectory on the road network. So, the partial sequential pattern is good feature candidate for creating model for trajectory classification.

Sun, G.Z [14] propose a model of a time warping recurrent neural network (TWRNN) to handle temporal pattern classification where severely time warped and deformed data may occur, and shows to have built-in time warping ability. TWRNN having advantages for trajectory classification over several schemes such as dynamic programming, hidden Markov models, time-delayed neural networks, and neural network finite automata. 
Stephen Gang Wu [15] employs Probabilistic Neural Network (PNN) with image and data processing techniques to implement general purpose automated leaf recognition for plant classification. The system automatically classifies 32 kinds of plants via the leaf images loaded from devices such as digital cameras or scanners. PNN is having fast speed on training and has simple structure.

A. Fehske [16] proposed A New Approach to Signal Classification Using Spectral Correlation and Neural Networks. It has suggested a method for classifying the communication signals based on cyclic spectral analysis and pattern recognition performed by a neural network.

Guobin $\mathrm{Ou}$ [17] focused on Multiclass neural learning i.e. Multiclass Pattern Classification which involves finding appropriate neural network architecture, encoding schemes, learning algorithms. Discussion takes place on the major approaches used in neural networks for classifying multiple classes takes place and which architecture suitable for system of multiple neural networks or a single neural network.

There are various classification techniques such as Decision Tree, Rule Based Classification, Memory Based Classification, Support Vector Machine and Neural Network etc. used for building various classification model which work on data sets such as text, graphs, networks, patterns and trajectories on road network. Neural Network is in less use for creating model for trajectory classification as compared to SVM [18].

\section{PROPOSED SYSTEM}

In proposed System, we use neural network to build classification model. We extract Feature Vectors from set of trajectories. In extraction process we use sequential patterns. Feature vectors along with adjusted initial weight vectors are input to our model. The Figure 1 shows the Overall Learning Process using delta learning rule.

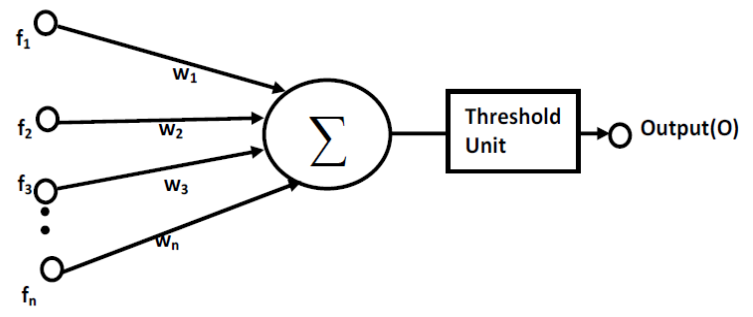

Fig 1 Learning Process of Neural Network

$\mathrm{O}$ is output which indicates class label. Threshold Unit is for threshold which is experimentally determined. Output is derived from following formula

$$
\operatorname{Output}(O)=\sum_{i=0}^{n} f i . w i
$$

Where, $F$ is the set of feature vector, $F=\left\{f_{1}, f_{2}, f_{3}, f_{4} \ldots f_{n}\right\}$. W is the set of weight vector, $\mathrm{W}=\{\mathrm{w} 1, \mathrm{w} 2, \mathrm{w} 3, \mathrm{w} 4 \ldots \mathrm{wn}\}$.

$\Delta \mathrm{W}$ checks how the weight changes during learning process which follows given formula $\Delta \mathrm{W}=(\mathrm{O}-\mathrm{Oi})$. Where, $\mathrm{Oi}$ is the expected Output. We will find out weight change using given formula i.e. $\mathrm{W}=\mathrm{W}+\Delta \mathrm{W}$.

Figure 2 shows Architecture of Supervised Learning Process in Neural Network. Supervised learning is a problem solving strategy which is driven by real business problem of history data and the Quality of result depend upon the quality of input data sets

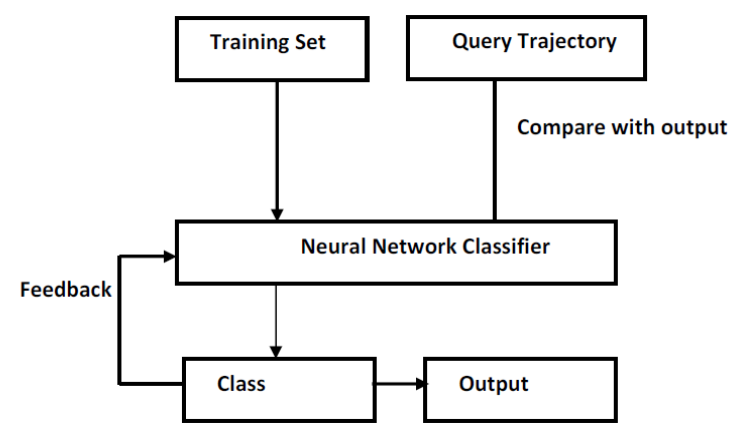

Fig 2 Architecture of Supervised Learning Process

In Figure 2, training set includes feature vector extracted from set of trajectories. Query Trajectory is for validate the result of classifier. Neural network classifier plays important role in classification process which produces output in the form of class. The class is changes in the training process as per the feedback.

\subsection{Proposed Algorithm :}

Input: Set of Feature Vector.

Output: Classification.

Training:

1. Input feature for training extracted from data set.

2. Input feature set to neural network.

3. Compare output (i.e. class) with expected output.

4. Provide feedback.

5. Adjust weight vector.

6. Go to step 2 and repeat procedure till expected output.

Classification:

1. Input feature for training extracted from data set.

2. Input feature set to neural network.

3. Output compare with actual output to find out accuracy.

\section{DISCUSSION AND FUTURE WORK}

We have proposed a model for classifying the trajectories on road network using neural network. We have proposed Algorithm which follows supervised learning process. We use Neural Network (NN) classifier because $\mathrm{NN}$ is a powerful tool for self-learning, and it can be generalized the characteristics of resource variations by proper training. In future, we will employ this technique on Real Trajectory Data as well as finding solution on Data Uncertainty.

\section{REFERENCES}

[1] Jae-Gil Lee, Jiawei Han, Fellow,Xiaolei Li, and Hong Cheng, "Mining Discriminative Patterns for Classifying Trajectories on Road Networks",IEEE TRANSACTIONS ON KNOWLEDGE AND DATA ENGINEERING, VOL. 23, NO. 5, MAY 2011

[2] G. Gido falvi and T.B. Pedersen, Mining Long, Sharable Patterns in Trajectories of Moving Objects, GeoInformatica, vol. 13, no. 1, pp. 27-55, 2009. 724 IEEE TRANS-ACTIONS ON KNOWLEDGE AND DATA ENGINEERING, VOL. 23, NO. 5, MAY 2011 TABLE 8 The Summary of the Notation Fig. 15. Estimation of the information gain as $\mathrm{k}$ varies. 
[3] J. Gudmundsson and M.J. Kreveld, Computing Longest Duration Flocks in Trajectory Data, Proc. 14th ACM Intl Symp. Geographic Information Systems, pp. 35-42, Nov. 2006.K. Elissa, "Title of paper if known," unpublished.

[4] N. Mamoulis, H. Cao, G. Kollios, M. Hadjieleftheriou, Y. Tao, and D.W. Cheung, Mining, Indexing, and Querying Historical Spatiotemporal Data, Proc. ACM SIGKDD, pp. 236-245, Aug. 2004.

[5] Y. Zheng, L. Zhang, X. Xie, and W.-Y. Ma, Mining Interesting Locations and Travel Sequences from GPS Trajectories, Proc. 18th Intl Conf. World Wide Web, pp. 791- 800, Apr. 2009

[6] T. Brinkhoff, A Framework for Generating NetworkBased Moving Objects, GeoInformatica, vol. 6, no. 2, pp. 153-180, 2002

[7] R. Agrawal and R. Srikant, Mining Sequential Patterns, Proc. 11th Intl Conf. Data Eng., pp. 3-14, Mar. 1995.

[8] X. Yan, J. Han, and R. Afshar, "CloSpan: Mining Closed Sequential Patterns in Large Databases," Proc. Third SIAM Int'l Conf. Data Mining, May 2003.

[9] H. Lodhi, C. Saunders, J. Shawe-Taylor, N. Cristianini, and C. Watkins, "Text Classification Using String Kernels," J. Machine Learning Research, vol. 2, pp. 419444, 2002.

[10] C.S. Leslie, E. Eskin, and W.S. Noble, "The Spectrum Kernel: A String Kernel for SVM Protein classification," Proc. Seventh Pacific Symp. Biocomputing, pp. 566-575, Jan. 2002.

[11] M. Deshpande, M. Kuramochi, N. Wale, and G. Karypis, "Frequent Substructure-Based Approaches for Classifying Chemical Compounds," IEEE Trans. Knowledge and Data Eng., vol. 17, no. 8, pp. 10361050, Aug. 2005.
[12] L.R. Rabiner and B.H. Juang, "An Introduction to Hidden Markov Models," IEEE ASSP Magazine, vol. 3, no. 1, pp. 4-16, Jan. 1986.

[13] R. Fraile and S.J. Maybank, "Vehicle Trajectory Approximation and Classification," Proc. Ninth British Machine Vision Conf., pp. 832-840, Sept. 1998.

[14] Sun, G.Z.; Chen, H.H.; Lee, Y.C.; Liu, Y.D. Time warping recurrent neural networks and trajectory classification, Neural Networks, 1992. IJCNN. International Joint Conference. Volume: 1 Publication Year: 1992 , Page(s): 431 - 436 vol.1

[15] Stephen Gang Wu, Forrest Sheng Bao, Eric You Xu, YuXuan Wang, Yi-Fan Chang and Qiao-Liang Xiang, "A Leaf Recognition Algorithm for Plant Classification Using Probabilistic Neural Network", Signal Processing and Information Technology, 2007 IEEE International Symposium, pp. 11-16 Dec 2007.

[16] A. Fehske, J. Gaeddert and J. H. Reed, "A New Approach to Signal Classification Using Spectral Correlation and Neural Networks", New Frontiers in Dynamic Spectrum Access Networks, 2005, DySPAN 2005. 2005 First IEEE International Symposium on, Publication Year: 2005, Page(s): 144 - 150.

[17] Guobin Ou, Yi Lu Murphey, and Lee Feldkamp, "Multiclass Pattern Classification Using Neural Networks", Pattern Recognition, 2004. ICPR 2004. Proceedings of the 17th International Conference on (Volume: 4), pp. 585 - 588 Vol.4.

[18] C.-C. Chang and C.-J. Lin, LIBSVM: A Library for Support Vector Machines, http://www.csie.ntu.edu.tw/ cjlin/libsvm, 2001 